\title{
On the Value of Information in a Strategic Conflict
}

\author{
MORTON I. KaMieN \\ Kellogg Graduate School of Management, Northwestern University, \\ Evanston, Illinois 60208 \\ YAIR TAUMAN \\ Faculty of Management, Tel Aviv University, 69978 Tel Aviv, Israel
}

AND

SHMUel ZAMIR

Department of Statistics, Hebrew University, 91904 Jerusalem, Israel

Received August 8, 1988

\begin{abstract}
We consider a situation in which an agent $\mathbf{M}$ (the "maven") possesses information relevant to the players of an $n$-person game in which he is not a participant. We define the "inducible set" as the set of all outcomes which can be made unique Nash equilibria of a game resulting from the maven's transmission of information. This inducible set is a formal expression of M's ability to manipulate the game. We demonstrate some properties of the inducible set and characterize it for 2-person zero-sum games. Finally, we define the notion of the "value of information" possessed by $\mathrm{M}$ and provide an explicit formula to calculate this value in terms of the inducible set. Journal of Economic Literature Classification Number: 026. 1990 Academic Press, Inc.
\end{abstract}

\section{INTRODUCTION}

The presence of uncertainty imparts value to information. In a decision theoretic framework the value of information equals the increment in expected utility an individual can realize by possessing it (see Hirshleifer and Riley, 1979) and therefore is the most he would be willing to pay to acquire it. In determining the value of information the individual does not, 
in the decision theoretic framework, explicitly consider how the actions of others will affect it nor what information others possess. Neither feature remains valid in a general conflict situation under uncertainty involving more than one decision maker, namely, a game.

The question we address is: What is an appropriate definition of the "value of information" in an extensive form game? As in the decision theoretic framework this value should reflect how much the parties to the conflict are willing to pay for it. However, in the decision theoretic framework, the value of information can be determined independently of the mechanism by which payment takes place. This is not true in the game thcorctic framework because of the presence of externalities that give rise to the free rider problem. To deal with value of information in the context of a strategic conflict we posit the existence of a sole possessor of information, dubbed "the maven," who is not a party to the conflict, and ask how much he can profit by disclosing some or all of it to some or all the affected parties. The nonparticipation of the maven in the conflict is assumed so as to assure his impartiality beyond his quest to maximize the value of his information. We imbue him with sole possession of the information so as to abstract from the effects of competing mavens on its value. Finally, because it is commonly easier to sell something specific than something vague, we focus on those situations in which disclosure of information leads to an unambiguous outcome of the conflict situation. It should be kept in mind that the maven is merely an artifact for getting at the value of information. Moreover, our analysis is in the spirit of offering an approach to determining the value of information in any specific context rather than providing a value of information for all contexts. Our approach to this question requires resolution of the following two questions.

(a) Given a strategic conflict, which outcomes can the maven induce through partial or full disclosure of information to the players? The maven's ability to affect the outcomes may be regarded as his possession of "power" (as in the expression "knowledge is power").

(b) What profit can the maven realize from his power to induce changes in the conflict situation by various disclosures of information? This involves determining the optimal mode of its sale.

We begin by defining a natural "action space" for the maven, which is the space of possible information transmissions. It includes the naive actions of revealing or not revealing some information to a certain player and more sophisticated ones as well. The maven's actions are similar in nature to the communication devices proposed in Forges (1986) to study communication equilibria. In our context this is equivalent to an extensive form communication device, i.e., a communication device at each 
node of the tree. These communication devices have the special feature that the players have no input to send.

Next we describe the consequences of the maven's actions in the space of outcomes of the games: What outcomes can he induce using the actions available to him? We use a rather strong definition of this notion. The maven can induce a certain outcome if he has an action (i.e., information transmission) that makes it the unique perfect Nash equilibrium of the resulting game. The set of all such outcomes is called the inducible set, which we propose as an expression for the maven's ability to manipulate the game.

Despite the strong definition of inducibility adopted here, the inducible set may be "large" in nontrivial families of games of interest. We consider such a family and display properties of its inducible set. A full characterization of the inducible set of 2-person zero-sum games is also presented.

We then turn to the second question, attributing an economic value to the maven's power as measured by the inducible set. All mechanisms the maven can design to achieve a certain point in the inducible set are considered. The value of his information is defined to be the highest payoff, $v$, he can "guarantee" himself. Under an optimal mechanism, the maven collects the payoff $v$ from the players of the game. Furthermore, it is a dominant strategy for each player to pay his share in $v$. We provide an explicit formula (Theorem 7.2, below) to calculate the value of information. This result has been applied in Kamien et al. (1988) to determine the optimal licensing mechanism for a cost reducing innovation of significant magnitude.

The paper is structured as follows. In Section 2 we discuss the issue of information transmission via an example. We demonstrate a signaling strategy that motivates the definition and the results presented in Sections 3 and 4 . In Section 5 we apply the results of Section 4 to characterize the inducible set of the example of Section 2. In Section 6 we characterize the inducible set of any 2-person zero-sum game in extensive form. The economic value of information and the related literature are discussed in Section 7.

\section{An Example}

The following example is intended to fix ideas in clarifying the main issues and concepts and motivate the formal theory that follows. Consider the following three-stage game:

Stage 0. A black (B) or white (W) card is drawn by chance and placed face down. Each color has equal probability of bcing drawn. 


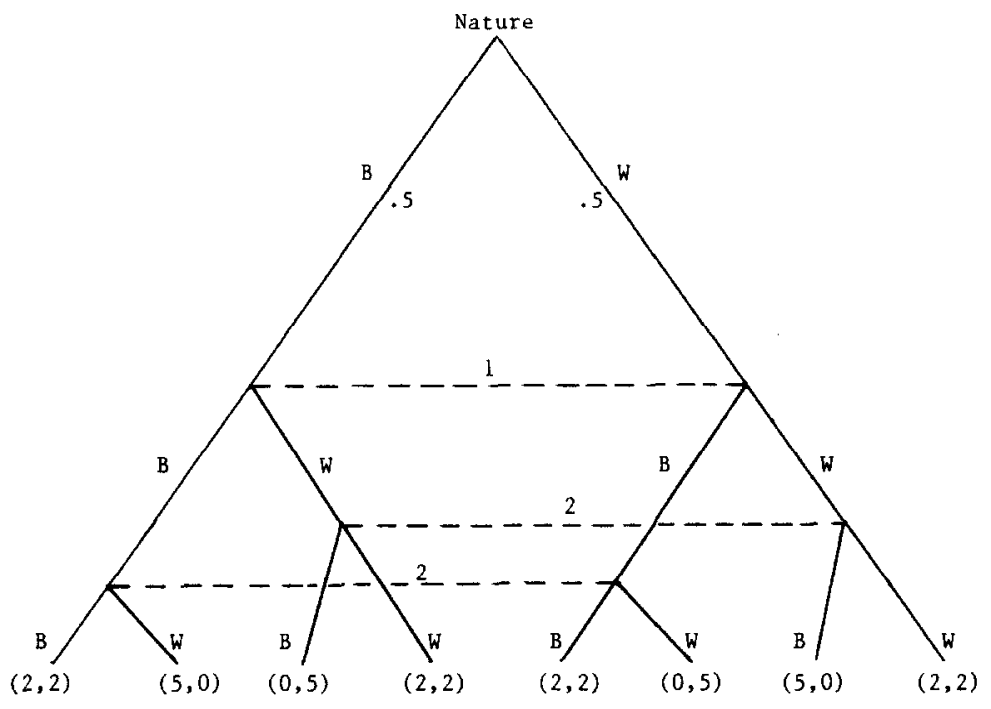

FIG. 1. The black and white game.

Stage 1. Player 1 announces a color B or W.

Stage 2. Player 2 knowing the color announced by player 1 announces a color B or W.

Payoffs: If both players announce the same color, each obtains 2 units. If the announced colors are different, the player who announced it correctly receives 5 while the other receives 0 .

Denote this game by $\Gamma\left(\frac{1}{2}\right)$ (parametrized by the probability of B in stage 0 ). The extensive form of the game is given in Fig. 1.

It is easily seen that this game has a unique Nash equilibrium (NE) outcome with expected payoff $(2.5,2.5)$. In fact it is a dominant strategy for player 2 to announce the color opposite to that announced by player 1 , since it yields an expected payoff $\frac{1}{2} \cdot 5+\frac{1}{2} \cdot 0=2.5$ which exceeds the sure payoff of 2 if he announces the same color.

Suppose now that a third party, $M$, the maven, knows the color of the card chosen at stage 0 . Although he is not a player in the original game, he has the ability to affect its outcome by disclosing his information. The obvious way of doing so is to inform either one or both players about the actual color of the card chosen in stage 0 . There are four possibilities.

If only player 1 is informed of the true color, and it is common knowledge that this is the case, the resulting game is described in Fig. 2. It is now a dominant strategy for player 1 to announce the true color and knowing that, the only best reply for player 2 is to repeat the same color, 


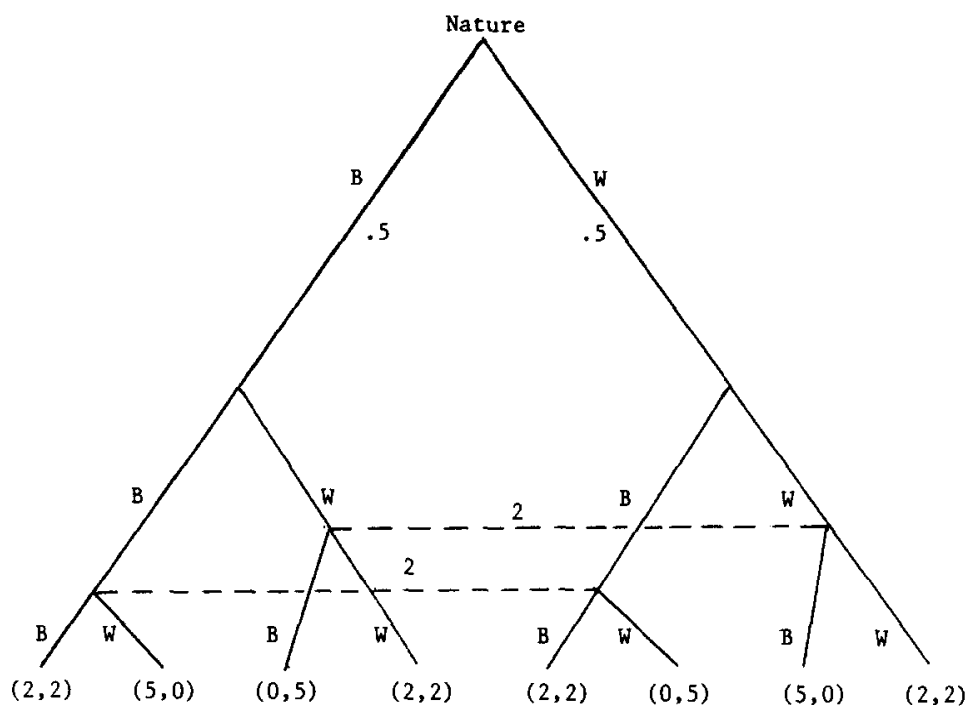

FIG. 2. It is common knowledge that only Player 1 is informed.

yielding (2.2) as the only NE outcome of the game. This outcome is strictly inferior for both players than the NE outcome of the original game. This means in particular that the informed player 1 is worse off than the uninformed player 1 of the original game. It is tempting to conclude that "information can be harmful" to a player, but it is not the information itself that harms player 1 but the fact that player 2 knew that he had it.

By the same argument one concludes that the game in which both players are informed also has $(2,2)$ as the unique NE outcome (since this is just a consequence of the common knowledge that player 1 is informed). Finally if only player 2 is informed of the true color it is a dominant strategy for him to announce the correct color and thus the only $\mathrm{NE}$ outcome is $.5(2,2)+.5(0,5)=(1,3.5)$.

The four simplistic possibilities of information transmission are summarized by their outcomes in Fig. 3 in what we call the $\mathrm{I}-\mathrm{U}$ matrix, in which I stands for "informed" and U for "uninformed."

Can the maven induce other outcomes? In particular can he induce a payoff above 3.5 to player 2? It may be thought that player 2's best situation is to be exclusively and fully informed as player 1 , who moves first, cannot take advantage of the information transmitted to player 2 . Yet it turns out that player $M$ can transmit "partial" information to the two players that will induce a payoff of $\frac{25}{7}$ to player 2 (and $\frac{10}{7}$ to player 1 ). Let us show this before formally describing the set of all strategies available to M. Player M's strategies consist of two parts. The first is a com- 
Player 2

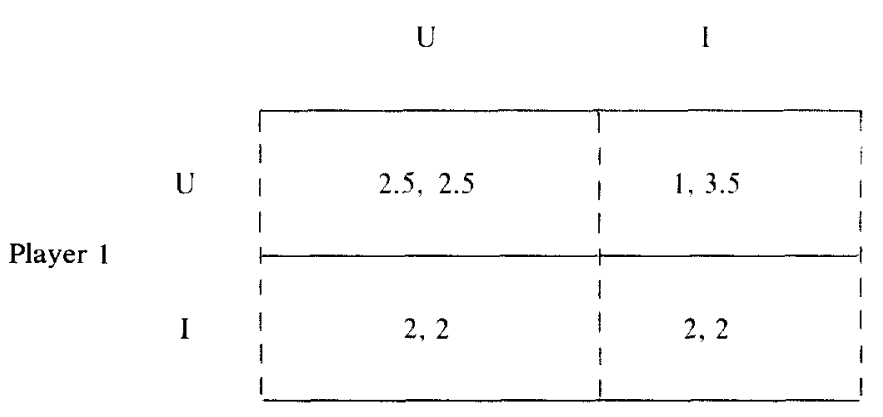

FIG. 3. The I-U matrix of the black and white game.

mon signal $\beta$ or $\omega$ transmitted to both players before player 1's move. The second is a private signal to player 2 after player 1's move. The common signal is chosen as follows: if the true color is $\mathrm{B}$, player $\mathrm{M}$ chooses $\beta$ or $\omega$ with probabilities $\frac{5}{7}$ and $\frac{2}{7}$, respectively. If the true color is $W$ the probabilities are $\frac{2}{7}$ and $\frac{5}{7}$, respectively. After player 1's move, player $M$ reveals the true color to player 2 only if player 1's move "matches" the signal announced by player $M$ (i.e., if player 1 chooses B following the announcement $\beta$ or chooses $W$ following $\omega$ ).

Suppose now that player M's above strategy, $\sigma$, becomes common knowledge to the two players. To describe the game $\Gamma_{\sigma}$ induced by $\sigma$, observe that

$$
\text { Prob (Player M announced } \omega)=\left(\frac{1}{2}\right)\left(\frac{5}{7}\right)+\left(\frac{1}{2}\right)\left(\frac{2}{7}\right)=\frac{1}{2}
$$

and by Bayes' rule

$$
\begin{aligned}
& \text { Prob (true color is } B \mid \text { player M announced } \omega \text { ) } \\
& =\left(\frac{2}{7}\right)\left(\frac{1}{2}\right) /\left[\left(\frac{2}{7}\right)\left(\frac{1}{2}\right)+\left(\frac{5}{7}\right)\left(\frac{1}{2}\right)=\frac{2}{7} .\right.
\end{aligned}
$$

Similarly,

Prob (true color is $B \mid$ player $M$ announces $\omega$ ) $=\frac{\pi}{7}$.

Thus, the game $\Gamma_{\sigma}$ can be described by Figs. 4, 5, and 6, where the subgames $\Gamma_{\beta}$ and $\Gamma_{\omega}$ are shown in Figs. 5 and 6.

Let us now find the perfect equilibrium point of $\Gamma_{\beta}$. Taking into account that player 2 chooses the correct color when he is informed about it, we 


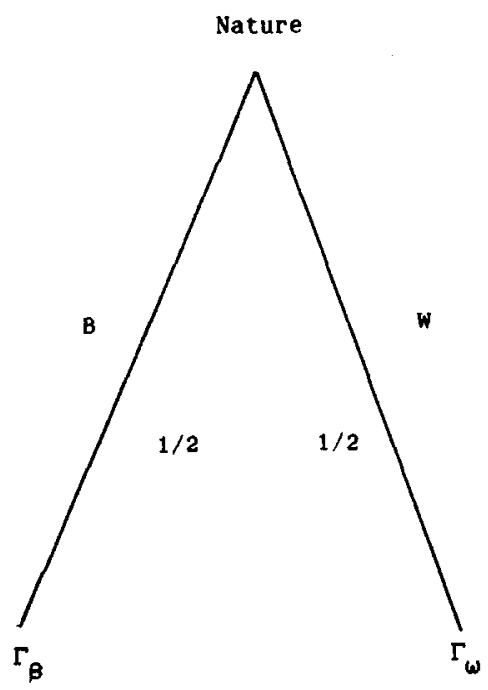

Figure 4

describe his strategy in $\Gamma_{\beta}$ by specifying his moves only when he is not informed about the correct color (i.e., when player 1 chooses W). Thus, we obtain the following strategic form game:

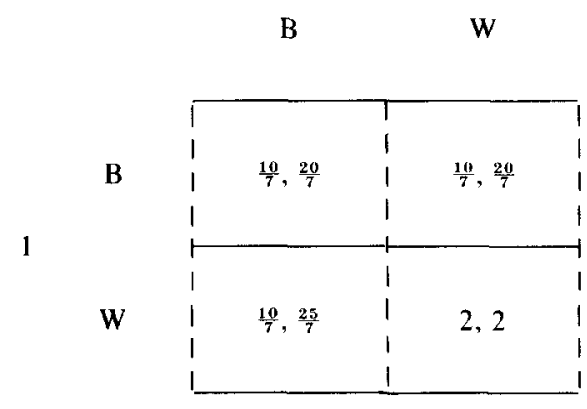

In this game player 1's choice of $\mathrm{W}$ and player 2's choice of $\mathrm{B}$ are dominant. Thus the unique perfect equilibrium payoff of $\Gamma_{\beta}$ is $\left(\frac{10}{7}, \frac{25}{7}\right)$. Similarly $\left(\frac{10}{7}, \frac{25}{7}\right)$ is also the unique perfect equilibrium payoff of $\Gamma_{\omega}$ and, hence, also of the game $\Gamma_{\sigma}$. It is interesting to note that in the game $\Gamma_{\beta}$ player 1 chooses $\mathrm{W}$ although his conditional probability of matching the true color 


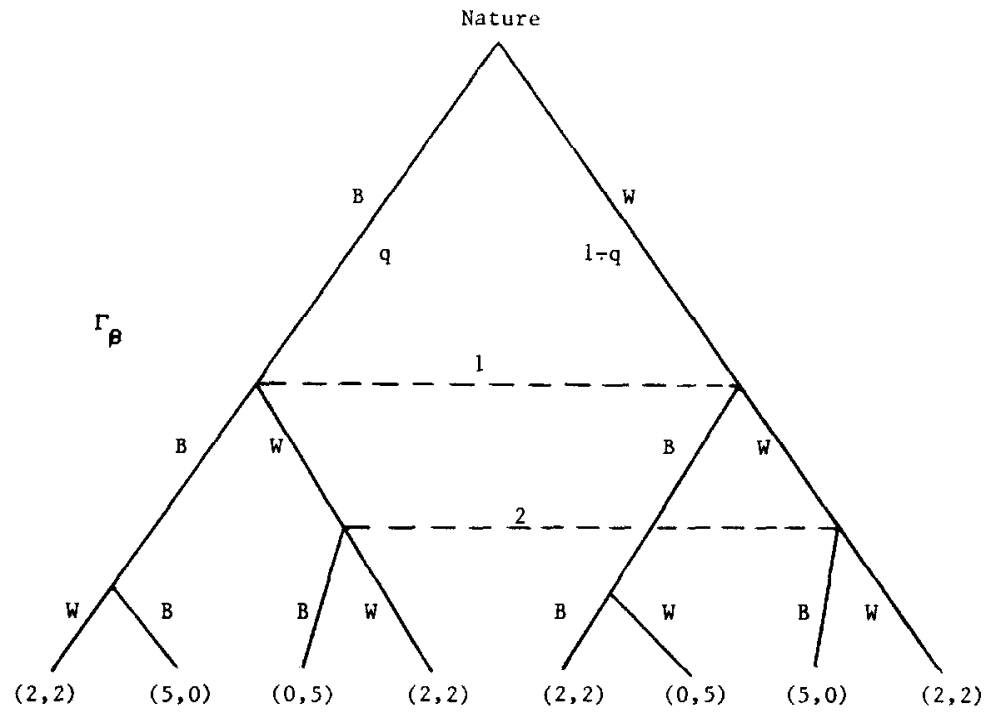

Figure 5

is only $\frac{2}{7}$ compared to $\frac{5}{7}$ if he would have chosen B. He does this to prevent player 2 from being informed about the true color. The set of all outcomes inducible by the maven in this example will be characterized in Section 5 where we employ the definitions and results of the next section.

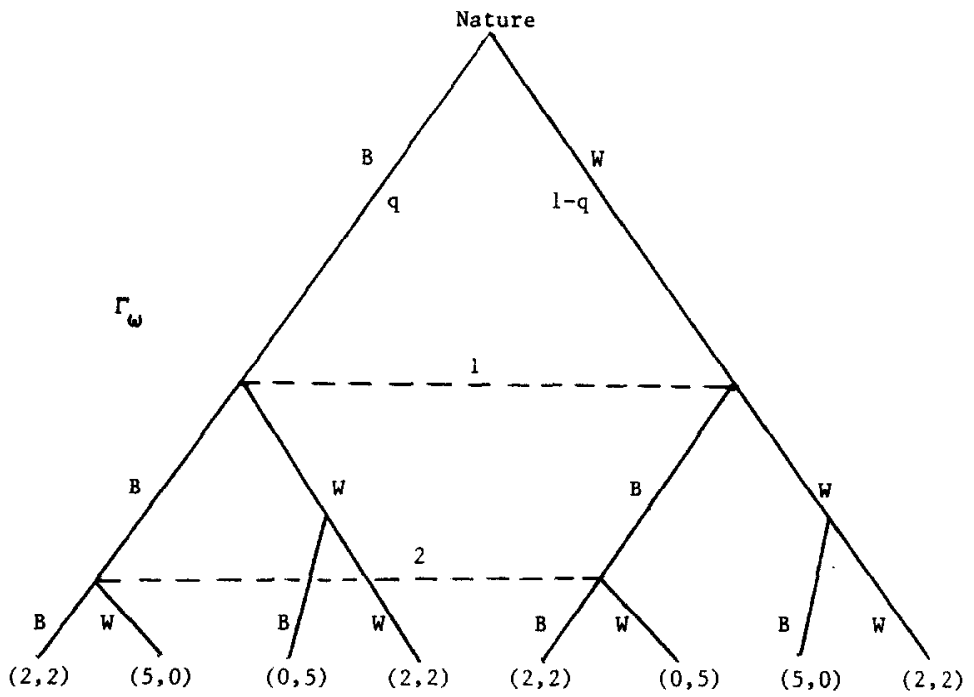

FIGURE 6 


\section{Signaling Strategies}

Let $\Gamma$ be a finite $n$-person game in extensive form with the set of players $N=\{1, \ldots, n\}$. Let $M \notin N$. We refer to $M$ as the maven or player $M$.

Definition 3.1. Player M's information is a partition $E$ of the set of all nonterminal nodes of $\Gamma$.

This definition provides considerable generality: player M's information may be about chance moves, about players' moves, or about any combination of the two. $E$ may be a refinement of a particular player's information sets, which means that player $M$ "knows more" than that player. In general, however, an information element $e \in E$ may intersect two of the same player's information sets. This means that player $M$ does not know some information known to that player. Furthermore, an element $e \in E$ may intersect two information sets of two different players $i$ and $j$. This means that player $\mathrm{M}$ at $e$ does not know which player's move it is but still has some information that may be relevant to one or both of them.

Definition 3.2. (1) Player M's signal set is a set $S$ of any alphabet.

(2) Player M's set of pure strategies is $\Sigma_{0}=\left(S^{N}\right)^{E}$ and the set of mixed strategies is $\Sigma=\Delta\left(\Sigma_{0}\right)$ (the set of probability distributions on $\Sigma_{0}$ ).

The signal set $S$ is the set of messages; each element of $S$ is a message that can be communicated by Player $M$ to each of the players. A pure strategy is a prescription of what message to send to each of the players in each information element $e \in E$. We denote a pure strategy $s \in \Sigma_{0}$ by $s=\{s(e)\}_{e \in E}$ with the interpretation that if the node $x \in e$ belongs to player $i$ then upon reaching it, he receives the $i$ th component of $s(e)$ before making his move.

We assume that a strategy $\sigma$ of player $\mathrm{M}$ becomes common knowledge before the beginning of the game and that he is committed to implementing it. This eliminates the possibility of player $M$ "cheating" by transmitting a signal which is not according to $\sigma$. Consequently, at any point in the induced game the players' beliefs are consistent in Harsanyi's sense; i.e., they are derived from common prior given their private information.

The above assumption also rules out the idea of "secret" transmission of information, e.g., to inform player 1 (in the black and white example) about the color while player 2 "thinks" that he is uninformed. Strictly speaking this is not a game; it is a situation in which player 2 "thinks" that he is playing the game in Fig. 1 while in fact he is playing the game in Fig. 2. Imbedding this in a larger game with incomplete information would necessarily involve inconsistent beliefs that are not possible in our model. 
Any strategy $\sigma \in \sum$ of player $\mathrm{M}$ modifies the original game $\Gamma$ to another game with the same set of players. We denote this game by $\Gamma_{\sigma}$ and call it the game induced by the strategy $\sigma$. If $\sigma_{0}$ is a pure strategy then $\Gamma_{\sigma_{11}}$ is obtained from $\Gamma$ by refining each player's information sets by the signals he receives from $M$. If $\sigma$ is the mixed strategy using pure strategies $\sigma_{0}^{1}, \ldots, \sigma_{0}^{k}$ with probabilities $p_{1}, \ldots, p_{k}$, respectively, then $\Gamma_{\sigma}$ is the extensive form game shown in Fig. 7. The "information set" in Fig. 7 indicates that for each of the players the same nodes in $\Gamma_{\sigma_{\theta}^{j}}$ and $\Gamma_{\sigma_{k}^{l}}$ are indistinguishable unless the signals received there are distinct.

DeFINITION 3.3. (i) A payoff vector $x \in R^{N}$ is inducible by player M in the game $\Gamma$ if there exists a $\sigma \in \Sigma$ such that the induced game $\Gamma_{\sigma}$ has a unique perfect Nash equilibrium point with payoff $x$.

(ii) The inducible set in $\Gamma$ with respect to information $E$ is the closure of all payoffs inducible by player $\mathrm{M}$ with information $E$. (Notation: $X=X(\Gamma, E)$.)

Note that the uniqueness of $\sigma$ is not required; a certain $x \in X$ may be inducible by two (or more) different strategies $\sigma$ and $\sigma^{\prime}$. In this case each of $\Gamma_{\sigma}$ and $\Gamma_{\sigma^{\prime}}$ has a unique perfect NE with payoff $x$.

Remark. Definition 3.3 is a special case of a conceivably more general definition of inducibility. Let $\mathrm{G}$ be a subset of the class of all games in extensive form with a given set $N=\{1, \ldots, n\}$ of players. Given a game

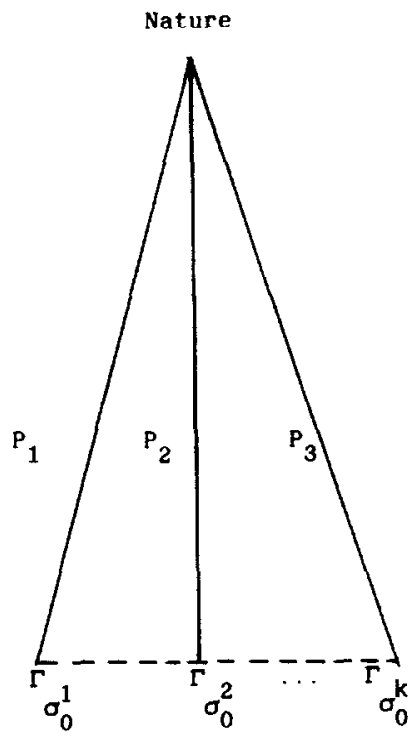

FIGURE 7 
$\Gamma$ with the set of player $N$ and given the information partition $E$ we define

$$
\Gamma_{E}=\left\{G \in \underset{\sim}{\mathrm{G}} \mid \exists \sigma \in \Sigma, \text { s.t. } G=\Gamma_{\sigma}\right\} .
$$

Let $\phi$ be a single valued solution concept $\phi: G \rightarrow \mathbb{R}^{n}$. The inducible set $X$ of $\Gamma$ with respect to $\phi$ is defined to be the closure of the image $\phi\left(\Gamma_{E}\right)$. In Definition 3.3, G is the set of all games with unique perfect $\mathrm{NE}$ and $\phi$ associates with each game in $\mathrm{G}$ the payoff of its perfect NE.

Lemma 3.4 For any $\Gamma$ and any $E$, the set of all inducible outcomes is convex.

Proof. Let $x=\lambda_{1} x_{1}+\cdots+\lambda_{k} x_{k}$, where $x_{i} \in X, \lambda_{i}>0 ; i=1, \ldots$, $k$ and $\sum \lambda_{i}=1$. Let $\sigma_{i} \in \Sigma$ be such that $x_{i}$ is the payoff for the unique NE in $\Gamma_{\sigma_{i}}$. Let $\sigma$ be the strategy in which player $M$ chooses $\left\{\sigma_{i}\right\}_{i=1}^{k}$ with probabilities $\left\{\lambda_{i}\right\}_{i=1}^{k}$, announces $\sigma_{i}$ to all players, and then implements it. It is readily seen that $\Gamma_{\sigma}$ has a unique NE with payoff $x$.

COROLlary. The noninducible outcomes in $X$ can be only on the boundary of $X$.

\section{Information about Chance Moves}

A class of games of special interest is that in which player $\mathbf{M}$ knows the outcomes of some of the chance moves in $\Gamma$. By taking the product space of all chance moves known to player $M$ we may equivalently consider the situation in which there is one chance move with outcomes $O=$ $\left\{o_{1}, \ldots, o_{k}\right\}$ ("states"), probability distribution $p=\left(p_{1}, \ldots, p_{k}\right)$, and the chosen state is known to player $M$. We view $p$ as a parameter of the game with range $\Delta(O)=\left\{p=\left(p_{1}, \ldots, p_{k}\right) \in R^{k} \mid p_{i} \geq 0, \sum p_{i}=1\right\}$, and write $\Gamma(p), \Gamma_{\sigma}(p)$, etc.

Lemma 4.1 (Splitting strategy). Let $\lambda=\left(\lambda_{1}, \ldots, \lambda_{m}\right), \lambda_{j} \geq 0$, $\sum \lambda_{j}=1$, and let $p,\left(p^{j}\right)_{j=1}^{m}$ be points in $\Delta$ such that $p=\sum_{j=1}^{m} \lambda_{j} p^{j} ;$ then in $\Gamma(p)$ player $M$ can induce a game which is equivalent to the following: a chance move chooses a member of $\left\{p^{1}, \ldots, p^{m}\right\}$ according to the probabilities $\lambda_{1}, \ldots, \lambda_{m}$, all players are informed of the outcome $p^{j}$, and then $\Gamma\left(p^{j}\right)$ is played.

This is a well known result from the theory of games with incomplete information (see Mertens and Zamir, 1971, Lemma 2, p. 46). We provide a rather simple proof for the sake of completeness.

Proof. Let $S_{0}=\left\{s_{1}, \ldots, s_{m}\right\}$ be any subset of $m$ signals from $S$. For $i \in\{i, \ldots, k\}$ for which $p_{i}>0$ let $\gamma^{i}=\left(\gamma_{1}^{i}, \ldots, \gamma_{m}^{i}\right) \in \Delta\left(S_{0}\right)$ be de- 
fined by $\gamma_{j}^{i}=\lambda_{j} p_{i}^{j} / p_{i} ; i=1, \ldots, k, j=1, \ldots, m$. Consider the following signaling strategy by player $\mathrm{M}$ : if the true state is $o_{i}$, perform the lottery $\gamma^{i}$ on $S_{0}$ (i.e., for $j=1, \ldots, m$, choose signal $s_{j}$ with probability $\gamma_{j}^{i}$ ) and announce the outcome to all players. The probability of announcement $s_{j}$ is $\Sigma_{i} p_{j} \gamma_{j}^{i}=\Sigma_{i} \lambda_{j} p_{i}^{j}=\lambda_{j}$, and for $\lambda_{j}>0$ the conditional probability on $O$ given announcement $s_{j}$ is

$$
P\left(\text { State is } o_{i} \mid \text { announcement is } s_{j}\right)=p_{i} \gamma_{j}^{i} / \lambda_{j}=p_{i}^{j} \text {. }
$$

In other words, the conditional distribution on $O$ given the announcement $s_{j}$ is $p_{j}$. The resulting game is therefore equivalent to the one in Fig. 8 , completing the proof of the lemma.

Let $X: \Delta \rightarrow 2^{R^{n}}$ be the set valued function from $\Delta$ to subsets of $R^{n}$ where $X(p)$ is the inducible set of $\Gamma(p)$. The set $X(p)$ may be empty for some or even for all $p \in \Delta$ since by our definition inducibility has to occur via a unique NE. Consider, for instance, a case as in Fig. 9 in which chance chooses one of two games $\Gamma_{\mathrm{L}}$ and $\Gamma_{\mathrm{R}}$ with probabilities $p, 1-p$, respectively, and the chosen game is publicly announced and played. In such a game player $\mathrm{M}$ has no role at all if $p=0$ or $p=1$. Therefore if, say, $\Gamma_{\mathrm{L}}$ has more than one equilibrium payoff then $X(1)=\varnothing$. However, Lemma 3.4 states that whenever $X(p) \neq \varnothing$ it is convex. A consequence of Lemma 4.1 is that the set $D=\{p \in \Delta \mid X(p) \neq \varnothing\}$ is also convex. Actually the same argument demonstrates a stronger result, namely:

THEOREM 4.2. The graph of the set valued function $X$ defined on $D$ is a convex set in $\Delta \times R^{n}$.

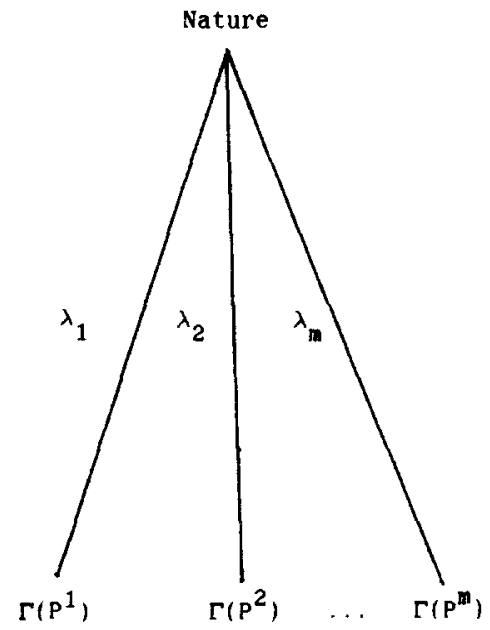




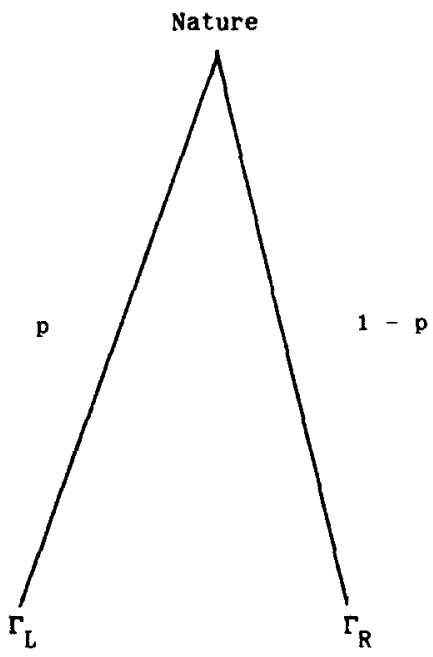

FIGURE 9

Proof. Let $x_{1} \in X\left(p^{1}\right), x_{2} \in X\left(p^{2}\right) ; p=\lambda p^{1}+(1-\lambda) p^{2} ; 0 \leq \lambda$ $\leq 1$. We have to show that $x=\lambda x_{1}+(1-\lambda) x_{2} \in X(p)$. If $p^{1}=p^{2}$ or $\lambda=0$ or $\lambda=1$, the result follows from Lemma 4.1, so assume $p^{1} \neq p^{2}$, and $0<\lambda<1$.

For any $\varepsilon>0, x_{1} \in X\left(p^{1}\right)$ implies that $\exists \sigma_{1} \in \sum$ such that $\Gamma_{\sigma_{1}}\left(p^{2}\right)$ has a unique NE with payoff $\varepsilon$-close to $x_{1}$. Similarly $\exists \sigma_{2} \in \sum$ such that $\Gamma_{\sigma_{2}}\left(p^{2}\right)$ has a unique NE with payoff $\varepsilon$-close to $x_{2}$. Let $\bar{\sigma}$ be the mixed strategy inducing (by Lemma 4.1) the game of Fig. 10.

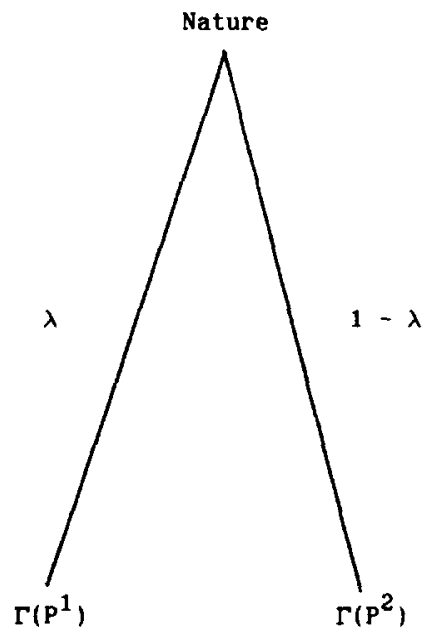

Figure 10 


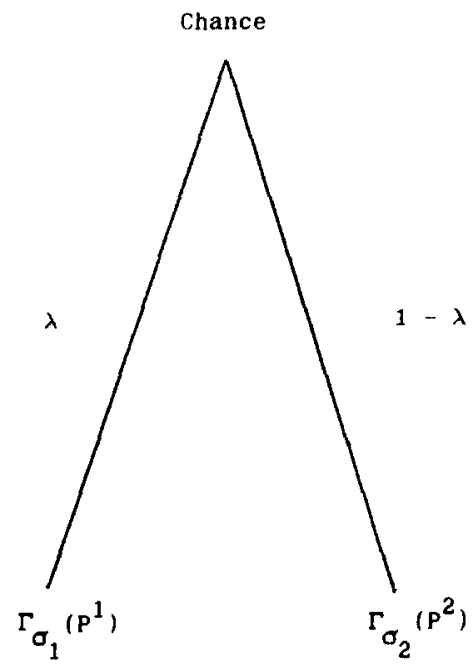

FiguRE 11

Let $\sigma$ be player M's strategy consisting of $\tilde{\sigma}$ followed by $\left(\sigma_{1}, \sigma_{2}\right)$, that is: perform the lottery $\tilde{\sigma}$, announce the outcome 1 or 2 , and then apply $\sigma_{1}$ or $\sigma_{2}$, respectively. Clearly this can be written as a signaling strategy (mixed or behavioral) based on the signal set $\{1,2\} \times S$. The resulting game is equivalent to the one of Fig. 11, and it is common knowledge to all players whether 1 or 2 is reached. Clearly the unique $\mathrm{NE}$ of this game is the one which follows the unique NE in $\Gamma_{\sigma_{1}}\left(p^{1}\right)$ or $\Gamma_{\sigma_{2}}\left(p^{2}\right)$ according to whether the state is 1 or 2 . The corresponding payoff is $\lambda x_{1}$ $+(1-\lambda) x_{2}=x$. Therefore $x \in X(p)$, completing the proof of the theorem.

It is worth mentioning that $x(p)$ is continuous on int $D$, where $D=\{p \in \Delta \mid x(p) \neq \varnothing\}$. This follows from the next lemma which is proved in the Appendix.

Lemma 4.3. Let $\tilde{C}$ be a convex subset of $\mathbb{R}^{m}$ and let $f$ be a single valued function from $\tilde{C}$ into subsets of $\mathbb{R}^{k}$. Suppose that the graph of $f$ is convex on $\tilde{C} \times \mathbb{R}^{k}$ and that $f(x)$ is a closed subset of $\mathbb{R}^{k}$ for each $x \in C$. Then $f$ is continuous in Int $\tilde{C}$ (the interior of $\tilde{C}$ ).

\section{The Black and White Game Revisited}

Let us now find the inducible set for the black and white game $\Gamma\left(\frac{1}{2}\right)$. Thus far we have shown the following: 
- The payoff $(2,2)$ is inducible by informing player 1 (or both players) of the color of the card.

- The payoff $\left(1, \frac{7}{2}\right)$ is inducible by informing player 2 only.

- Payoffs arbitrarily close to $\left(\frac{10}{7}, \frac{25}{7}\right)$ are inducible by the behavioral signaling strategy described in Section 2.

Assume that player $\mathrm{M}$ has full information both about nature's moves and player 1's move, i.e., $E=\{\{0\}\{1\},\{2\},\{3\},\{4\},\{5\},\{6\}\}$ (see Fig. 5).

Proposition 5.1. $(3,2)$ belongs to the inducible set $X\left(\Gamma\left(\frac{1}{2}\right), E\right)$.

Proof. Consider the game $\Gamma(p)$ for $0 \leq p \leq 1$. Its strategic form is given by

BB

BW

WB

WW

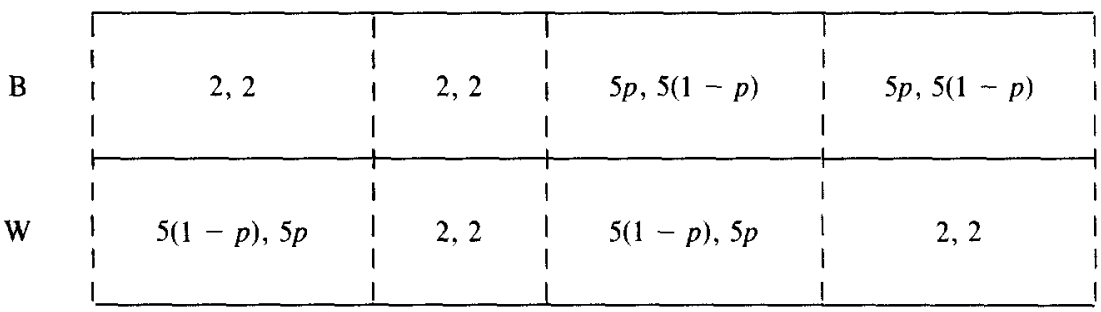

Here the left component of player 2's strategy indicates his move if player 1 announces $B$ and the right component indicates his move if player 1 announces W. For $p=\frac{2}{5}+\varepsilon$ the game $\Gamma(p)$ has a unique perfect equilibrium in which player 1 announces W and player 2 plays WB (which is the opposite choice to that of player 1). The resulting payoff is $(3-5 \varepsilon$, $2+5 \varepsilon)$. Similarly the game $\Gamma\left(\frac{3}{5}-\varepsilon\right)$ has a unique perfect equilibrium point which results in the same payoff, $(3-5 \varepsilon, 2+5 \varepsilon)$. Consequently $(3-5 \varepsilon, 2+5 \varepsilon) \in X\left(\frac{2}{5}+\varepsilon\right) \cap X\left(\frac{3}{5}-\varepsilon\right)$. Thus, by Theorem $4.2,(3-5 \varepsilon$, $2+5 \varepsilon) \in X\left(\frac{1}{2}\right)$ for each $\varepsilon$. Since $X\left(\frac{1}{2}\right)$ is a closed set, $(3,2) \in X\left(\frac{1}{2}\right)$ and the proof is complete.

Note that this proof does not establish the inducibility of $(3,2)$. In fact, we suspect that $(3,2)$ is not inducible although we do not have a proof for that.

Proposition 5.2. The inducible set in $\Gamma\left(\frac{1}{2}\right)$ with information $E=\{\{0\}$, $\{1\},\{3\},\{4\},\{5\},\{6\}\}$ for player $M$ is (see Fig. 12)

$$
X\left(\frac{1}{2}\right)=\operatorname{Con}\left\{(2,2),(3,2),\left(1, \frac{7}{2}\right),\left(\frac{10}{7}, \frac{25}{7}\right\},\right.
$$

where for a set $S$, Con $S$ denotes the convex hull of $S$. 


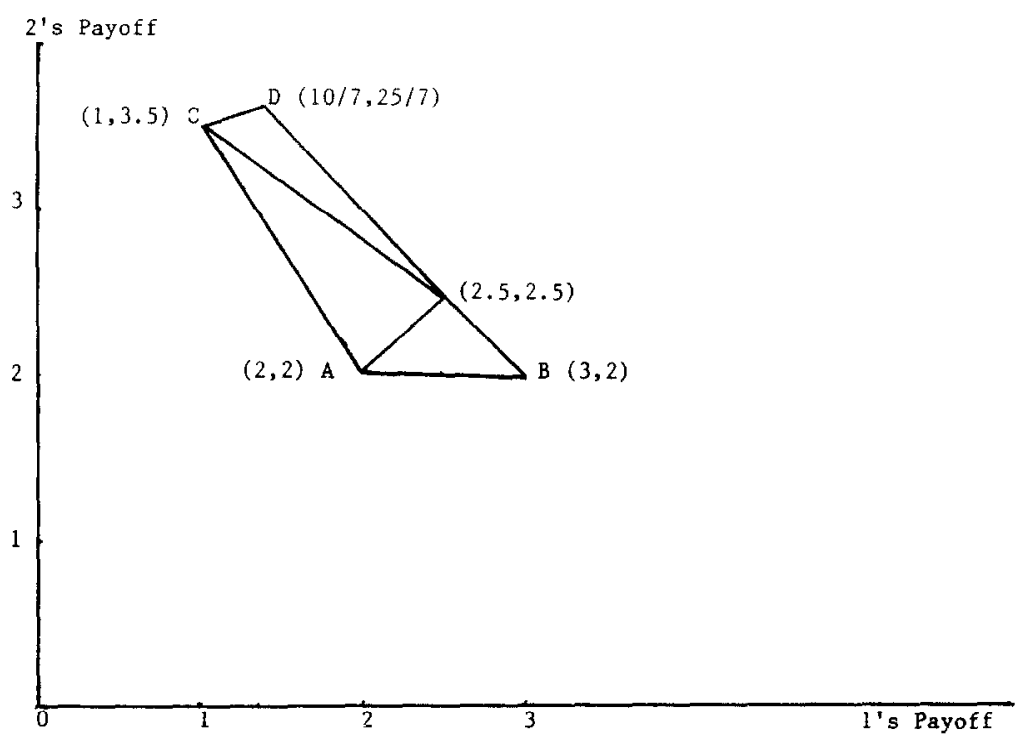

FIG. 12. The inducible set for the black and white game.

Proof. We have already shown that all four points, $A=(2,2)$, $B=(3,2), C=\left(1, \frac{7}{2}\right)$, and $D=\left(\frac{10}{7}, \frac{25}{7}\right)$, are in the inducible set. It follows from Proposition 3.4 that

$$
\operatorname{Con}\{A, B, C, D\} \subset X\left(\frac{1}{2}\right) .
$$

To prove the inverse inclusion it is sufficient to show that any inducible outcome $\left(x_{1}, x_{2}\right)$ is constrained by the appropriate four straight lines, that is:
(i) $x_{1}+x_{2} \leq 5$
(line BD)
(ii) $3 x_{1}+2 x_{2} \geq 10$
(line $A C)$
(iii) $x_{2} \geq 2$
(iv) $x_{1}-6 x_{2}+20 \geq 0 \quad$ (line $\mathrm{CD}$ ).

Constraints (i) and (ii) are satisfied since these are constraints on the set of all possible outcomes, which is $\operatorname{Con}\{(2,2),(5.0),(0,5)\}$. Constraint (iii) is clearly satisfied since 2 is player 2 's minimax value in any $\Gamma_{\sigma}$ : player 2 can guarantee it simply by repeating player 1's announcement. Consequently no NE of $\Gamma_{\sigma}$ (for any $\sigma$ ) yields player 2 a payoff smaller than 2 . It is therefore sufficient to prove that (iv) is satisfied for any $\mathrm{NE}$ of $\Gamma_{\sigma}$. To see this write any possible payoff $\left(x_{1}, x_{2}\right)$ as a convex combination:

$$
\left(x_{1}, x_{2}\right)=\alpha(0,5)+\beta(5,0)+(1-\alpha-\beta)(2,2) .
$$


Observe first that if (iv) is violated for $\left(x_{1}, x_{2}\right)$ then

$$
4 \alpha>3 \beta+2 .
$$

Assume now that $\left(x_{1}, x_{2}\right)$ satisfying $(*)$ and $(* *)$ is in the inducible set. Then there is a $\sigma \in \Sigma$ and $(f, g)$, and NE strategies of players 1 and 2 in $\Gamma_{\sigma}$ with the corresponding payoff $\left(x_{1}, x_{2}\right)$ satisfying $(*)$ and $(* *)$.

The information sets of player 1 in $\Gamma_{\sigma}$ are partitioned into three types:

- $U_{1}^{\mathrm{B}}$ (resp. $U_{1}^{\mathrm{W}}$ ) information in which player 1 knows for sure (among other things) that the color is B (resp.W).

- $U_{1}^{0}$ information set in which player 1 does not know for sure whether the color is $\mathrm{B}$ or $\mathrm{W}$.

Given $\sigma, f$, and $g$, at any information set $U_{1}$ of player 1 , let $(\tilde{\alpha}, \bar{\beta},(1-\tilde{\alpha}$ $-\tilde{\beta})$ ) be the conditional probabilities of outcomes $(0,5),(5,0)$, and $(2,2)$ at $U_{1}$. Since by assumption, the (unconditional) probabilities $(\alpha, \beta)$ satisfy $(*)$ and $(* *)$, there must be at least one information set $U_{1}$ reached (by $(f, g))$ with positive probability, for which the probabilities $(\tilde{\alpha}, \tilde{\beta})$ satisfy

$$
4 \tilde{\alpha}>3 \tilde{\beta}+2 .
$$

$(* * *)$

Consider such a $U_{1}$. It cannot he of type $U_{1}^{\mathrm{B}}$ or $U_{1}^{\mathrm{W}}$ since in those sets $\bar{\alpha}=0$ (since player 1 can avoid $(0,5)$ ). Assume that (according to $f$ ) player 1 chooses $\mathrm{W}$ at $U_{1}$ (the other case treated in the same way), and let $\gamma$ be the (conditional) probability, at $U_{1}$, that the true state is $\mathrm{B}$. When choosing $\mathrm{W}$, the outcome $(0,5)$ can be obtained only if the state is $\mathrm{B}$. Hence $\gamma \geq \tilde{\alpha}$. So by choosing $\mathrm{W}$ (according to $f$ ) at $U_{1}$ player 1 expects: $0 \bar{\alpha}+5 \bar{\beta}+2(1-\tilde{\alpha}-\bar{\beta})$, but if he deviates and plays $\mathrm{B}$ he expects at least $2 \gamma$ (with probability $\gamma$ it is the correct color and he gets at least 2). Now $2 \gamma \geq 2 \tilde{\alpha}$, and $(* * *)$ is equivalent to $2 \tilde{\alpha}>5 \tilde{\beta}+2(1-\tilde{\alpha}-\tilde{\beta})$. This is a contradiction to the fact that $(f, g)$ is a NE.

This completes the proof of Proposition 5.2.

Note again that the inducible set we established for the black and white game is strictly larger than the convex hull of payoffs in the I-U matrix (see Fig. 9).

The signaling strategies proposed here provide the maven a broad action space because he can induce outcomes not reachable by $\mathrm{I}-\mathrm{U}$ actions alone and he can even sometimes induce a selection of a specific $N E$ point from a set of several. This is illustrated in the following example.

EXAMPLE 5.3. Consider a situation in which a chance move allows, with equal probabilities, one of two matrix 2-person games, $G_{\mathrm{L}}$ and $G_{\mathrm{R}}$, and play occurs without knowledge of chance's choice (see Fig. 13). If 


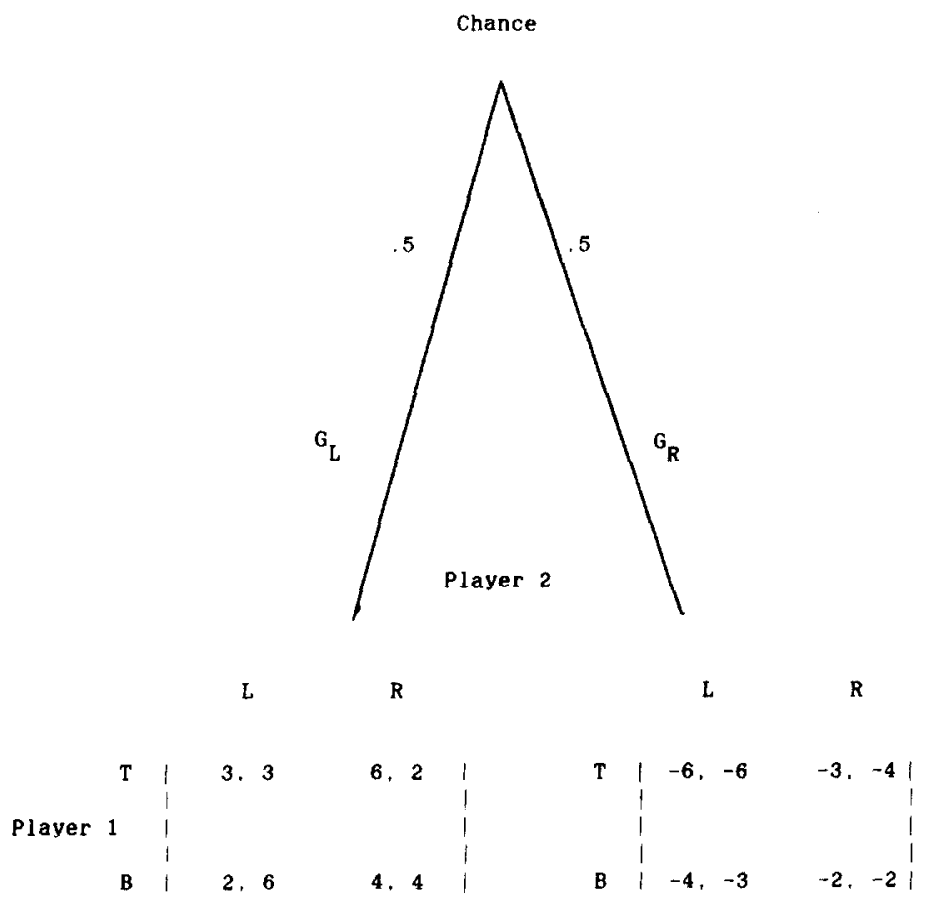

FIG. 13. The game of Example 5.3.

player M, knowing which game is actually being played, restricts his actions to inform/not inform, the NE of the resulting induced games are given by

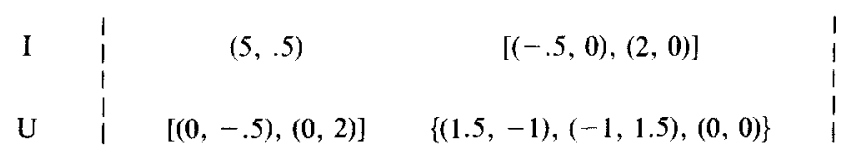

The feature to be noted here is that except for the case in which neither player is informed (i.e., the original game), all the other three games have multiple NE; if only one player is informed, there is an entire segment of NE payoffs and if both are informed there are three NE. So with our definition of inducibility, it would appear that only $(0.5,0.5)$ is inducible by the I-U actions. However, with the above defined general signaling we have the following result proved by Kamien et al. (1988): 
Proposition 5.4. The inducible set $X$ of the game in Example 5 satisfies (see Fig. 14)

$$
X \supset \operatorname{Con}\{(2,0),(0,2),(1.5,-1),(-1,1.5),(-1,0),(-1,0),(0,-1)\} .
$$

In Fig. 14 the polyhedron $A B C D E F G$ is the set of all possible outcomes (i.e., payoffs for 1 and 2) which are of the form $x=.5 x_{\mathrm{L}}+.5 x_{\mathrm{R}}$, where $x_{\mathrm{L}}$ is a point in the convex hull of $\{(3,3),(6,2),(2,6),(4,4)\}$ and $x_{\mathrm{R}}$ is a point in the convex hull of $\{(-6,-6),(-3,-4),(-4,-3),(-2,-2)\}$. The polyhedron ABCRSG is the convex hull of all Nash equilibrium payoffs in the $\mathrm{I}-\mathrm{U}$ matrix and strictly larger than it, is the polyhedron $\mathrm{ABCMNG}$, each point of which is inducible by the maven.

The fact that the I-U payoffs are in the inducible set mean that, for instance, $(1,0)$, which is onc point in the continuum of equilibria of the game in which only player 1 is informed, can be made the unique $N E$ in a game induced by some signaling strategy.

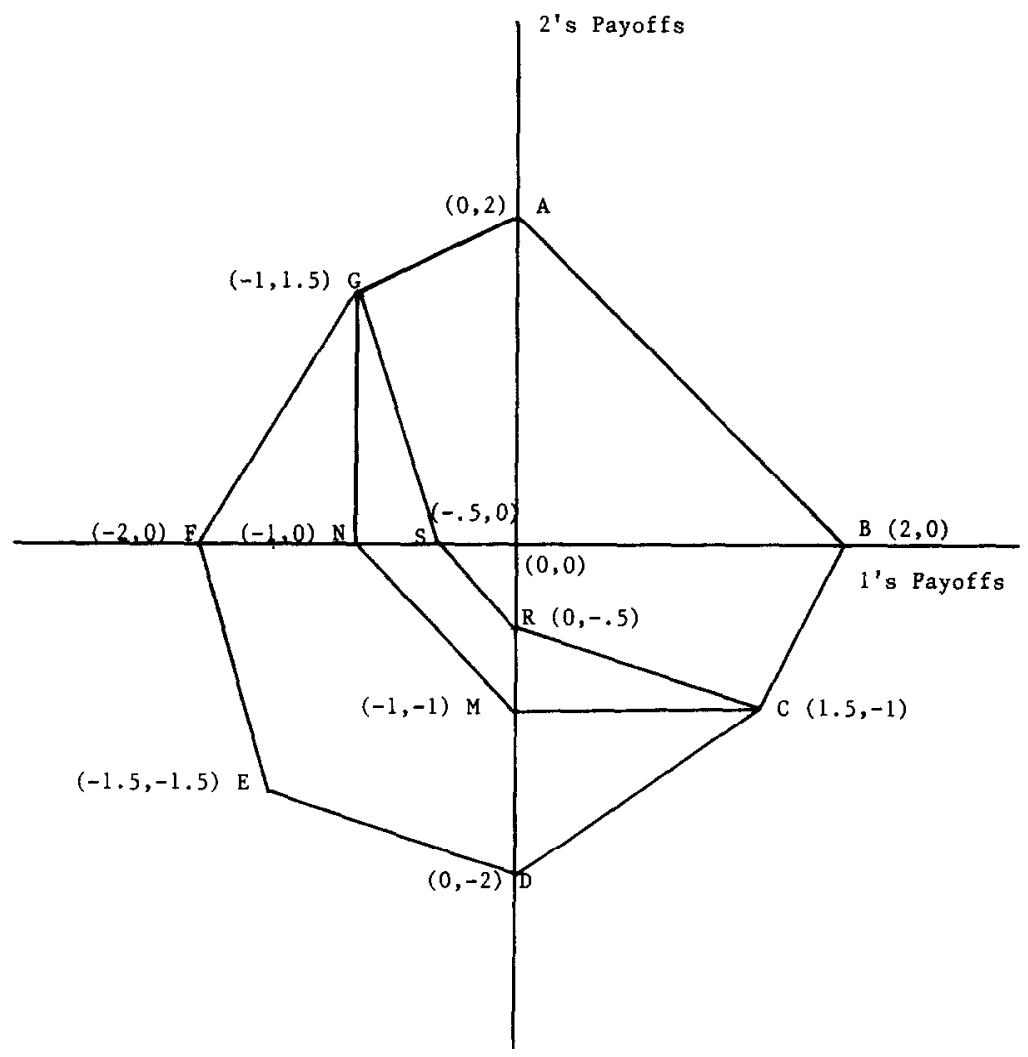

FIg. 14. The inducible set for the game in Example 4.5. 


\section{The 2-Person Zero-Sum Case}

In this section we treat the special case in which the original game $\Gamma$ is a 2-person, zero-sum game. We will confirm the well known statement that information has a positive value in this case: the best a maven can do for one player is to disclose it all to him and none to his opponent. The fact the opponent is aware of this does not matter and no sophisticated signaling can do better than that.

To state this result formally, let $\Gamma$ be a 2-person, zero-sum game. Let $E=\left\{e_{1}, \ldots, e_{m}\right\}$ player M's information. Take $S=\{0,1,2, \ldots, m\}$ to be the set of signals and define as before the set $\sum$ of (mixed) signaling strategies. For each $\sigma \in \Sigma$ denote, as usual, by $\Gamma_{\sigma}$ the game induced by $\sigma$ and its value by $v_{\sigma}$. Let $\bar{\sigma}$ and $\underline{\sigma}$ be the (pure) strategies of player M given by

$$
\widetilde{\sigma}\left(e_{i}\right)=(i, 0), \quad \underline{\sigma}\left(e_{i}\right)=(0, i), \quad \forall e_{i} \in E .
$$

with the interpretation that according to $\sigma$ : at $e_{i}$ communicate $i$ to player 1 and 0 (the neutral signal) to player 2 . Similarly for $\underline{\sigma}$.

THEOREM 6.1. $v_{\underline{\sigma}} \leq v_{\sigma} \leq v_{\bar{\sigma}}, \quad \forall \sigma \in \Sigma$.

That is, the inducible set is the segment $\left[v_{\sigma}, v_{\bar{\sigma}}\right]$.

Proof. Given any mixed strategy $\sigma$ of player M, define $\ddot{\sigma}$ by

$$
\tilde{\sigma}\left(e_{i}\right)=\left(i, \sigma_{2}\left(e_{i}\right)\right), \quad i=1,2, \ldots, m .
$$

Clearly in $\Gamma_{\bar{\sigma}}$ player I's strategy set contains his strategy set in $\Gamma_{\sigma}$ while the strategy set of player II is the same in both games. Since the values are maximin it follows that $v_{\sigma} \leq v_{\tilde{\sigma}}$. Observing that $\bar{\sigma}\left(e_{i}\right)=\left(\tilde{\sigma}_{1}\left(e_{i}\right), 0\right), i=1$, $2, \ldots, m$, it follows by the same arguments that $v_{\bar{\sigma}} \leq v_{\bar{\sigma}}$ and hence $v_{\sigma} \leq v_{\bar{\sigma}}$. Similarly one obtains $v_{\sigma} \leq v_{\sigma}$.

\section{The Value of INFormation}

In this concluding section we turn briefly to the question of what is the value of information to the maven. There is a vast literature dealing with the value of information both in economics and game theory. We refer to only a small subset of it. The relationship between the value of information to its possessor and the number of others having the information was analyzed by Hirshleifer (1971) in the context of the return to inventive activity, by Hakansson et al. (1982), and by Novos and Waldman (1982). Ponssard (1976) analyzed the implications of differences in information between duopolistic firms, while Sakai (1985) has also included the effects 
of these differences on consumers. Green and Stokey (1981) studied the value of information in the context of the principal-agent problem. Allen (1986) studied the value of information to consumers in a general equilibrium framework. Levine and Ponssard (1977) compared the values of public, private, and secret information to the players involved in a game of incomplete information. However, they did not consider the strategic behavior of the owner of information in assessing the values of the different types of information. Analyses of the value of information when its owner behaves strategically have been conducted by Kamien and Tauman (1984, 1986), Kamien et al. (1988), and Katz and Shapiro (1985, 1986) in the context of patent licensing, and by Admati and Pfleiderer (1986a,b), in the context of stock market information. Guth (1984) and Muto (1986) analyze the dissemination of information regarding a superior technology as it is resold by its initial purchasers.

The value of information to player $M$ derives from his ability to affect the game, that is, from the inducible set. The question we address is: Given a set $X \subset \mathbb{R}^{N}$ (to be interpreted as a set of payoff vectors to the players $N=\{1, \ldots, n\}$ ), and a player $M \notin N$ with the ability to induce any $x \in X$, what is the value of this power? However, at present, we do not have a completely satisfactory answer. In what follows we make the additional crucial assumption that player $M$ has the ability to design the mechanism of his negotiation with the set $N$ of players affected by his power (that is, a game with player $N \cup\{M\}$ ), and commit himself to implementing it. In such mechanisms, player M's only possible action is to choose some $x \in X$ and the reactions of the other players are to influence his choice by paying him (assuming, of course, the possibility of transferring utility from each player to player $\mathrm{M}$ ).

Definition 7.1.

(i) Player $\mathrm{M}$ can guarantee a payoff $z$ if for each $\varepsilon>0$ there is a mechanism with a unique NE in which it is a dominant strategy for each player $i \in N$ to pay $\alpha_{i}$, such that $\sum_{i \in N} \alpha_{i}=z-\varepsilon$.

(ii) The value of player M's power is the maximum payoff he can guarantee.

THEOREM 7.2. Given an inducible set $X$, the value of player $M$ 's power is given by

$$
v=\sup \left\{\sum_{i=1}^{n} x_{i} \mid\left(x_{1}, \ldots, x_{n}\right) \in X\right\}-\sum_{i=1}^{n} \inf \{x \mid x \in X\} .
$$

Proof. Assume for simplicity that all inf and sup are attained on $X$ and let $x^{*} \in \arg \max \sum x_{j}$... For $j \in N$ denote $\mu_{j}=\min \left\{x_{j} \mid x \in X\right\}$, $x^{j} \in\left\{x \in X \mid x_{j}=\mu_{j}\right\}$. Observe first that $M$ cannot guarantee more than $v$. Indeed, any payoff, $y_{M}$, of $\mathrm{M}$, is of the form 


$$
y_{M}=\sum_{i \in N} x_{i}-\sum_{i \in N} y_{i},
$$

where $x \in X$ and $y_{i}$ is the net payoff of $i$. Since $\sum_{i \in N} x_{i} \leq \sum_{i \in N} x_{i}^{*}$ and $y_{i} \geq \mu_{i}$ (individual rationality) we conclude that $y_{M}<v$ in any Nash equilibrium.

Consider now the following two-step mechanism:

Step 1. Player $\mathrm{M}$ requests that each player $j \in N$ pay him the amount $x_{j}^{*}-\mu_{j}-\varepsilon / n$. Each player can choose either to pay or not to pay. Let $N_{\mathrm{I}}=\{j \in N \mid j$ chooses not to pay $\}$.

Step 2. If $N_{1}=\varnothing$ player $\mathrm{M}$ induces $x^{*}$. If $N_{\mathrm{l}} \neq \varnothing$ player M gives each $j \in N \backslash N_{1}$ a refund of $x_{j}^{*}-\mu_{j}$ and induces $x^{k}$, where $k$ is the least index in $N_{1}$.

It is readily verified that in this mechanism it is a dominant strategy for each player $j \in N$ to pay $x_{i}^{*}-\mu_{j}-\varepsilon / n$. As a result, $x^{*}$ will be induced and player M's payoff will be $\Sigma_{j} x_{j}^{*}-\Sigma_{j} \mu_{j}-\varepsilon$.

Let us illustrate this result for the black and white game. A "best" outcome is $x^{*}=(2.5,2.5)$ which is obtained when player M does nothing. The individuals' minimum utility levels are

$$
\begin{array}{ll}
\mu_{1}=1 & \text { (in }\left(1, \frac{7}{2}\right) ; \\
\mu_{2}=2 & \text { (in }(2,2)) .
\end{array}
$$

So the value of player M's power, which in this case is the value of the information, is $v=2.5+2.5-1-2=2$. The mechanism which $\varepsilon$-achieves it is:

Step 1. Player 1 is asked to pay $1.5-\varepsilon / 2$. Player 2 is asked to pay $0.5-\varepsilon / 2$.

Step 2. The maven acts according to the reaction of the two players according to the following table:

Player 2

\begin{tabular}{cll} 
& Pay & \multicolumn{1}{c}{ Does not pay } \\
\hline Pay & Do nothing. & $\begin{array}{l}\text { Refund of } 1.5 \text { to player } 1 \\
\text { and announce publicly the } \\
\text { color of the card. }\end{array}$
\end{tabular}

Player 1

Does not pay Refund 0.5 to player 2 
It is easily verified that in the induced game it is a dominant strategy for both players to pay, giving the maven the total payoff of $2-\varepsilon$.

\section{APPENDIX}

Proof of Lemma 4.3. Lower semicontinuity follows directly from the convexity of the graph of $f$. It remains to prove that $f$ is upper semicontinuous on Int $C$. Let $x_{0} \in \operatorname{Int} C, x_{n} \rightarrow x_{0}, y_{n} \rightarrow y_{0}$, and $y_{n} \in f\left(x_{n}\right)$. Let us prove that $y_{0} \in f\left(x_{0}\right)$. Suppose to the contrary that $y_{0} \notin f\left(x_{0}\right)$. Since $f\left(x_{0}\right)$ is convex and closed, by the separation theorem there is a linear functional $\phi$ on $\mathbb{R}^{k}$ such that

$$
\phi\left(y_{0}\right)>\sup _{y^{\prime} \in f\left(x_{0}\right)} \phi\left(y^{\prime}\right) \geq 0 .
$$

Let $z_{1}, \ldots, z_{k+1}$ be $k+1$ points in $C$ such that $x_{0} \in \operatorname{Int} \operatorname{Conv}\left(z_{1}, \ldots\right.$, $z_{k+1}$ ). Then for each $\varepsilon>0$ there exists an $n$ sufficiently large such that $x_{0}$ can be represented as a convex combination of the form

$$
x_{0}=\alpha x_{n}+\sum_{i=1}^{k+1} \alpha_{i} z_{i}
$$

where $\alpha, \alpha_{i} \geq 0, \alpha+\sum \alpha_{i}=1$, and $\alpha \geq 1-\varepsilon$.

Now since the graph of $f$ is convex

$$
f\left(x_{0}\right) \supseteq \alpha f\left(x_{n}\right)+\sum_{i=1}^{k+1} \alpha_{i} f\left(z_{i}\right)
$$

Thus,

$$
\sup _{y^{\prime} \in f\left(x_{0}\right)} \phi\left(y^{\prime}\right) \geq \phi\left(y^{\prime \prime}\right) \quad \text { for each } y^{\prime \prime} \in \alpha f\left(x_{n}\right)+\sum_{i=1}^{k+1} \alpha_{i} f\left(z_{i}\right)
$$

By the linearity of $\phi$ we have

$$
\sup _{y^{\prime} \in f\left(x_{0}\right)} \phi\left(y^{\prime}\right) \geq \phi\left(y_{n}\right)+\sum_{i=1}^{k ! 1} \alpha_{i} \phi\left(\bar{y}_{i}\right), \quad \bar{y}_{i} \in f\left(z_{i}\right) .
$$

Now fix $\bar{y}_{i} \in f\left(z_{1}\right)$ and let $\mu=\max \left\{\left|\phi\left(\bar{y}_{i}\right)\right| \mid i=1, \ldots, k+1\right\}$. Taking the limit when $n \rightarrow \infty$ we obtain

$$
\sup _{y^{\prime} \in f\left(x_{0}\right)} \phi\left(y^{\prime}\right) \geq \alpha \phi\left(y_{0}\right)-\varepsilon \mu \geq(1-\varepsilon) \phi\left(y_{0}\right)-\varepsilon \mu .
$$


Since this is true for any $\varepsilon>0$, we have

$$
\sup _{y^{\prime} \in f\left(x_{0}\right)} \phi\left(y^{\prime}\right) \geq \phi\left(y_{0}\right)
$$

contradicting $(*)$.

\section{REFERENCES}

Admati, A. R., ANd Pfleiderer, P. (1986a). "A Monopolistic Market for Information," $J$. Econ. Theory 39, 400-438.

Admati, A. R., AND Pfleiderer, P. (1986b). "Direct and Indirect Sale of Information," Graduate School of Business, Stanford University, R.P. No. 899.

Allen, B. (1986). "The Demand for (Differential) Information," Rev. Econ. Stud. 53, 311 323.

Forges, F. (1986). "An Approach to Communication Equilibria," Econometrica 54, 13751385.

Green, J. R., And Stokey, N. L. (1981). "The Value of Information in the Delegation Problem," H.I.E.R. Discussion Paper No. 776, Harvard University.

GuTH, W. (1984). "How to Sell Valuable Information," Discussion Paper, Universitat Köln.

Hakansson, N. H., Kunkel, J. G., and Ohlson, J. A. (1982). "Sufficient and Necessary Conditions for Information to Have Social Value in Pure Exchange," J. Finance 37, $1169-1181$

Hirshleifer, J. (1971). "The Private and Social Value of Information and the Reward to Inventive Activity," Amer. Econ. Rev. 61, 561-573.

Hirshleifer, J., AND RILEY, J. G. (1979). "The Analytics of Uncertainty and Information-An Expository Survey," J. Econ. Lit. 17, 1375-1421.

Kamien, M. I., and Tauman, Y. (1984). "The Private Value of a Patent: A Game Theoretic Analysis," J. Econ. Suppl. 4, 93-118.

Kamien, M. I., AND Tauman, Y. (1986). "Fees versus Royalties and the Private Value of a Patent," Quart. J. Econ. 101, 471-491.

Kamien, M. I., Tauman, Y., and Oren, S. (1988). “Optimal Licensing of Cost-Reducing Innovation," Working Paper, Northwestern University.

Kamien, M. I., Tauman, Y., And Zamir, S. (1988). "The Value of Information in a Strategic Conflict," Revision of CMSEMS Working Paper No. 717, Northwestern University.

Kamien, M. I., Tauman, Y., and Zang, I. (1988). "Optimal License Fees for a New Product," Math. Soc. Sci. 16, 77-106.

Katz, M. L., And Shapiro, C. (1985). "On the Licensing of Innovations," Rand .I. Fcon. 16, 504-520.

Katz, M. L., AND Shapiro, C. (1986). "How to License Intangible Property," Quart. J. Econ. 101, 567-589.

Levine, P., and Ponssard, J. P. (1977). "The Values of Information in Some Nonzero Sum Games," Int. J. Game Theory 6, 221-229.

Mertens, J. F., and Zamir, S. (1971). "The Value of Two-Person Zero-Sum Repeated Games with Lack of Information on Both Sides." Int. J. Game Theory 1, 39-64. 
Muto, S. (1986). "An Information Good Market with Symmetric Externalities," Econometrica 54, 295-312.

Novos, I., and Waldman, W. (1982). "Markets for 'Potentially' Nonexcludable Commodities: The Case of Information," Center for the Study of Organizational Innovation, Discussion Paper No. 140, University of Pennsylvania.

PonsSARD, J. P. (1976). "On the Concept of Value Information in Competitive Situations," Manage. Sci. 22, 739-747.

SakaI, Y. (1985). "The Value of Information in a Simple Duopoly Model," J. Econ. Theory 36, 36-54. 